(n)

\title{
Automated image analysis of a glomerular injury marker desmin in spontaneously diabetic Torii rats treated with losartan
}

\author{
Tetsuhiro Kakimoto', Kinya Okada ${ }^{2}$, Yoshihiro Hirohashi ${ }^{3,+}$, Raissa Relator $^{3}$, \\ Mizue Kawai ${ }^{4}$, Taku Iguchi ${ }^{4}$, Keisuke Fujitaka ${ }^{4}$, Masashi Nishio ${ }^{4}$, Tsuyoshi Kato ${ }^{3}$, \\ Atsushi Fukunari ${ }^{1}$ and Hiroyuki Utsumi ${ }^{1}$ \\ ${ }^{1}$ Safety Research Laboratories and ${ }^{2}$ Advanced Medical Research Laboratories, Research Division, \\ Mitsubishi Tanabe Pharma Corporation, Toda-shi, Saitama 335-8505, Japan \\ ${ }^{3}$ Faculty of Science and Engineering, Gunma University, Kiryu-shi, Gunma 376-8515, Japan \\ ${ }^{4}$ Pharmacology Research Laboratories II, Research Division, Mitsubishi Tanabe Pharma Corporation, Toda-shi, \\ Saitama 335-8505, Japan \\ ${ }^{\dagger}$ Y Hirohashi is now at Electrical Energy Systems, Graduate School of Engineering, Tohoku University, 6-3, Aoba, \\ Aramaki, Aoba-ku, Sendai 980-8579, Japan
}

\author{
Correspondence \\ should be addressed \\ to T Kakimoto \\ Email \\ Kakimoto.Tetsuhiro@ \\ mf.mt-pharma.co.jp
}

\begin{abstract}
Diabetic nephropathy is a major complication in diabetes and a leading cause of end-stage renal failure. Glomerular podocytes are functionally and structurally injured early in diabetic nephropathy. A non-obese type 2 diabetes model, the spontaneously diabetic Torii (SDT) rat, is of increasing preclinical interest because of its pathophysiological similarities to human type 2 diabetic complications including diabetic nephropathy. However, podocyte injury in SDT rat glomeruli and the effect of angiotensin II receptor blocker treatment in the early stage have not been reported in detail. Therefore, we have evaluated early stages of glomerular podocyte damage and the beneficial effect of early treatment with losartan in SDT rats using desmin as a sensitive podocyte injury marker. Moreover, we have developed an automated, computational glomerulus recognition method and illustrated its specific application for quantitatively studying glomerular desmin immunoreactivity. This state-ofthe-art method enabled automatic recognition and quantification of glomerular desminpositive areas, eliminating the need to laboriously trace glomerulus borders by hand. The image analysis method not only enabled assessment of a large number of glomeruli, but also clearly demonstrated that glomerular injury was more severe in the juxtamedullary region than in the superficial cortex region. This applied not only in SDT rat diabetic nephropathy but also in puromycin aminonucleoside-induced nephropathy, which was also studied. The proposed glomerulus image analysis method combined with desmin immunohistochemistry should facilitate evaluations in preclinical drug efficacy studies as well as elucidation of the pathophysiology of diabetic nephropathy.
\end{abstract}

\section{Key Words}

- automated image analysis

- SDT rat

- glomerulus

- desmin

- superficial glomeruli

- juxtamedullary glomeruli http://joe.endocrinology-journals.org DOI: 10.1530/JOE-14-0164
() 2014 Society for Endocrinology Printed in Great Britain
Published by Bioscientifica Ltd
Journal of Endocrinology (2014) 222, 43-51 


\section{Introduction}

The number of patients with renal disease is increasing worldwide (Williams 2010). Among them, the most crucial cause of renal failure is type 2 diabetes mellitus. Recently, many diabetic animal models have been used to elucidate the mechanisms of pathogenesis and complications in diabetes. The spontaneously diabetic Torii (SDT) rat is of increasing preclinical interest, because of its pathophysiological similarities to human type 2 diabetic complications (Sasase et al. 2013).

The SDT strain is a newly established, non-obese type 2 diabetic rat model, inbred from the Sprague-Dawley (SD) rat (Shinohara et al. 2000). Male SDT rats spontaneously develop glucose intolerance from 16 weeks of age and show hyperglycemia without obesity after 20 weeks of age. From 24 weeks on, SDT rats show proteinuria because of renal dysfunction (Ohta et al. 2007). Histopathologically, periodic acid-Schiff-positive mesangial matrix deposition is observed from 50 weeks of age and progresses with aging. Thus, SDT rats will be a useful model for investigating diabetic nephropathy and suitable for preclinical drug efficacy studies. However, podocyte injury in the early stages has not been sufficiently studied in SDT rat glomeruli (Ohta et al. 2007, Fujii et al. 2010, Kim et al. 2012).

Growing evidence has indicated that podocyte injury is central to progression of diabetic nephropathy (Wiggins 2007), occurring at the early stage of diabetic glomerular damage. Increased desmin immunostaining in glomerular podocytes has been widely utilized as a sensitive podocyte injury marker and observed in various rat models of glomerular disease, including Heymann nephritis, and puromycin aminonucleoside (PAN)-induced nephrotoxicity, as well as diabetic nephropathy, such as Zucker diabetic fatty rats and Otsuka Long-Evans Tokushima fatty (OLETF) rats (Floege et al. 1992, Hoshi et al. 2002, Herrmann et al. 2012, Sofue et al. 2012). Accordingly, the quantification of glomerular desmin as a podocyte injury marker would be quite important for quantitatively evaluating early glomerular disease, particularly in the field of preclinical drug efficacy studies as well as for the elucidation of the pathophysiology of diabetic nephropathy. However, desmin immunoreactivity is also observed in vascular smooth muscle and renal interstitium. Thus, to quantify glomerular desmin-positive area, we need to distinguish glomeruli from other kidney structures.

Although the quantification of glomerular desminimmunopositive area has been reported by a few authors (Herrmann et al. 2012), they examined only a limited number of glomeruli out of more than 100 in a given kidney specimen by manually tracing each glomerulus border to segregate glomeruli from other kidney structures. Considering the heterogeneity of glomerular injuries in renal cortex (Sofue et al. 2012), the limited number of glomeruli analyzed by hand could lead to arbitrary selection and improper results. Automatic segregation of glomeruli from other renal structures is quite challenging for most image analysis software, which mostly depend on color feature information for recognition of tissue structures, because the colors of glomeruli are similar to those of other renal structures. In this study, we have developed an automatic computational image analysis method that utilizes brightness gradient instead of color feature information. Brightness gradient could depict morphological characteristics of an object in general. Specifically, our method used the histogram of oriented gradients (HOG) descriptor, which was originally proposed in computer vision (Dalal \& Triggs 2005) as a feature vector in a machine learning approach, a linear support vector machine (SVM) (Schölkopf \& Smola 2002, Shawe-Taylor \& Cristianini 2004). The pretrained linear SVM classifier automatically detected glomeruli during the scan of a whole-kidney-section image with a detection window. This state-of-the-art method has enabled automatic recognition and quantification of glomerular desmin-immunopositive area, eliminating the need of laborious manual tracing of glomerulus borders.

In this study, we have developed the state-of-the-art automatic computational method and illustrated its specific application to objectively and rapidly quantify the increase in desmin immunoreactivity in SDT rat glomeruli in the early stages of diabetes. We also examined the ameliorative effect of early treatment with an angiotensin II receptor blocker (ARB), losartan, which reportedly had beneficial effects on diabetic nephropathy in type 2 diabetes patients (Brenner et al. 2001). We also studied the heterogeneity of glomerular damage in renal cortices of not only SDT rats, but also of rats with PANmediated nephrosis, a toxicity model with selective podocyte injury.

\section{Materials and methods}

\section{Animals}

Male SD and SDT rats were purchased from Charles River Laboratories Japan (Yokohama, Japan) and CLEA Japan (Tokyo, Japan) respectively. The animals were acclimatized

Published by Bioscientifica Ltd 
for 1 week before study and housed under a $12 \mathrm{~h}$ light: $12 \mathrm{~h}$ darkness cycle with free access to water and chow. All animal experiments were performed in accordance with the institutional guidelines and approved in advance by the Committee of Animal Experiments of the Research Division of Mitsubishi Tanabe Pharma Corporation.

At 8 weeks of age, nine SDT rats, which were randomly divided, were administered losartan potassium (Wako, Osaka, Japan) in their drinking water at $40-50 \mathrm{mg} / \mathrm{kg}$ per day for 16 weeks. At 24 weeks of age, timed $24 \mathrm{~h}$ urine was collected using individual metabolic cages from five SD rats, ten SDT rats, and nine losartan-treated SDT rats, whereafter they were killed by exsanguination under anesthesia.

For the PAN model, 6-week-old SD rats received injections of $100 \mathrm{mg} / \mathrm{kg}$ of PAN (Sigma-Aldrich) via a tail vein and killed by exsanguination under anesthesia on day 4 or 7 (four animals each). In addition, four SD rats received injections of saline vehicle to provide controls.

\section{Plasma glucose and urinary albumin}

Blood samples were collected from a subclavian vein and the plasma fraction was separated by centrifugation. Plasma glucose levels were determined by glucose test (Wako) according to the manufacturer's instructions. Urinary albumin levels were determined using the Rat Albumin ELISA Kit (Shibayagi, Gunma, Japan) according to the manufacturer's instructions.

\section{Immunohistochemistry}

Kidneys removed from killed animals were immediately fixed in 10\% neutralized buffered formalin and embedded in paraffin for histological analysis. Immunohistochemistry was carried out as described previously (Kakimoto et al. 2013). Briefly, kidney paraffin sections were deparaffinized. The sections were incubated overnight at $4{ }^{\circ} \mathrm{C}$ with anti-desmin mouse MAB (Dako, Glostrup, Denmark), followed by HRP-conjugated secondary antibody (anti-mouse IgG goat polyclonal antibody; Nichirei, Tokyo, Japan). The sections were stained with $3,3^{\prime}$-diaminobenzidine and counterstained with hematoxylin. The sections were photographed using a BX51 microscope (Olympus, Tokyo, Japan) equipped with a DP73 digital camera system (Olympus) and cellSens Software (Olympus).

\section{Image analysis}

Whole-slide digital images of the immunostained sections were obtained with Aperio Scan Scope XT (Leica
Microsystems, Wetzlar, Germany). We have developed a novel computational method to automatically detect glomeruli and to quantify the glomerular immunopositive area. Briefly, a linear SVM classifier, which was pretrained using 512-dimensional HOG features, detected glomeruli in whole-slide images during scanning with a $200 \times 200$-pixeled detection window. Next, boundaries of the detected glomeruli were automatically determined using standard image processing, including edge detection, smoothing, binarization, and morphology operation, followed by the quantification of immunopositive area. At least 75 glomeruli were automatically recognized and analyzed for each rat. Detailed methods are available in the Supplementary Materials and Methods, see section on supplementary data given at the end of this article.

For analysis of superficial and juxtamedullary glomeruli, glomeruli located within a fixed distance from kidney surface were defined as superficial glomeruli, as described previously (Sofue et al. 2012). The remaining glomeruli located at the inner renal regions were classified as juxtamedullary glomeruli.

\section{Statistical analysis}

Results were expressed as mean \pm s.E.M. For the analysis of urinary albumin levels, differences between groups were calculated by nonparametric Kruskal-Wallis one-way ANOVA and Steel test. For other experiments, differences between groups were calculated by one-way ANOVA with post hoc Dunnett's test. The differences between groups were considered to be statistically significant when $P<0.05$.

\section{Results}

\section{Body weight, plasma glucose, and urinary albumin}

The body weight of SDT rats was significantly less than that of SD control rats at 24 weeks of age (Fig. 1A). Diabetes developed at this time, as SDT rats displayed overt hyperglycemia at 24 weeks of age (Fig. 1B). Losartan treatment yielded no significant difference in body weight of SDT rats and did not have a significant effect on plasma glucose of SDT rats (Fig. 1A and B).

SDT rats at 24 weeks of age showed significant albuminuria (Fig. 1C). Treatment with losartan lowered the mean value of the urinary albumin excretion of SDT rats, although the difference was not statistically significant.

Published by Bioscientifica Ltd. 

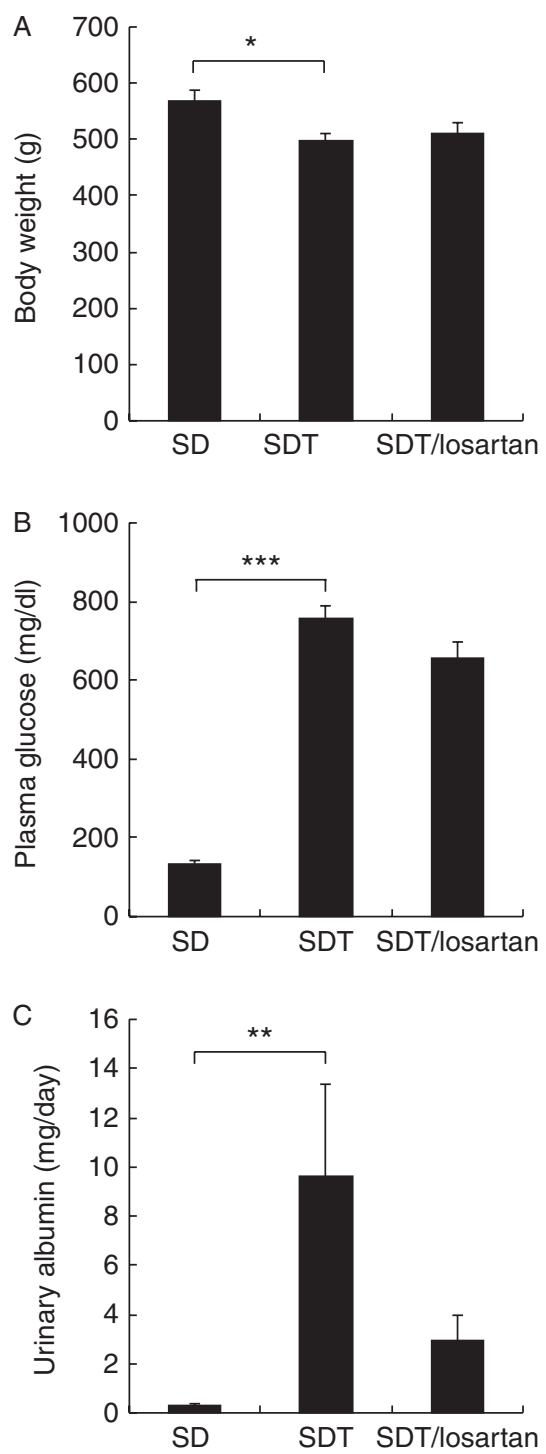

Figure 1

Body weight, plasma glucose, and urinary albumin. Body weight (A), plasma glucose concentration (B), and urinary albumin levels (C) of non-diabetic control SD rats and diabetic SDT rats at 24 weeks of age. Statistically significant differences are indicated by $* P<0.05, * * P<0.01$, and $* * * P<0.001$.

\section{Histopathology}

SD and SDT rats showed no obvious glomerular sclerosis at 24 weeks of age (data not shown), in accordance with a previous report (Ohta et al. 2007). Desmin immunohistochemistry revealed sparse staining in glomeruli of SD rats (Fig. 2A). In contrast, SDT rats displayed an obvious increase in desmin immunoreactivity (Fig. 2B). Losartan treatment visibly decreased desmin immunoreactivity in SDT glomeruli (Fig. 2C).

\section{Automated image analysis of glomerular desmin-immunopositive area in SDT rat kidneys}

To analyze quantitatively glomerular desmin immunoreactivity, we developed a novel computational method for determining glomerular desmin-immunopositive area.

\section{Desmin}
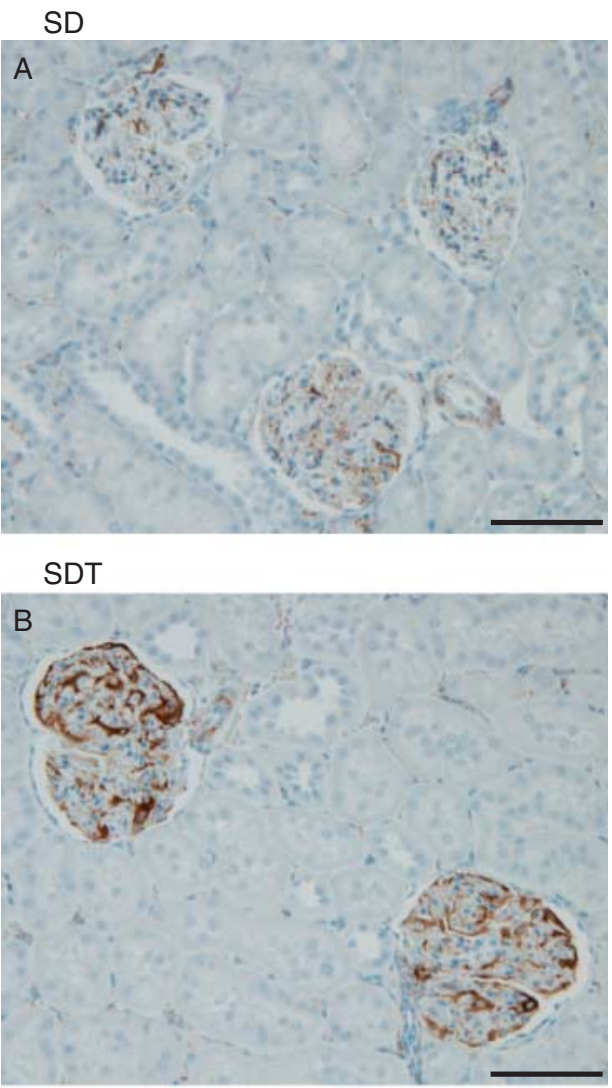

SDT/losartan

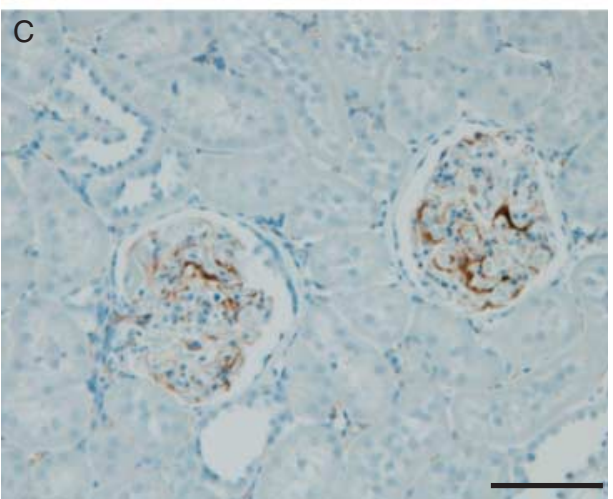

Figure 2

Glomeruli immunostained for desmin from 24-week-old SD (A), SDT (B), and losartan-treated SDT rats (C), all at 24 weeks of age. Sections were immunostained for desmin using the 3,3'-diaminobenzidine-based staining method and hematoxylin counterstaining. Scale bars: $100 \mu \mathrm{m}$.

Published by Bioscientifica Ltd. 
In our method, a detection window scanned the wholekidney section image and cropped candidate sub-images (Fig. 3A). To predict whether a glomerulus exists in the center of the cropped sub-image, a HOG feature vector (Dalal \& Triggs 2005) extracted from the sub-image was

A

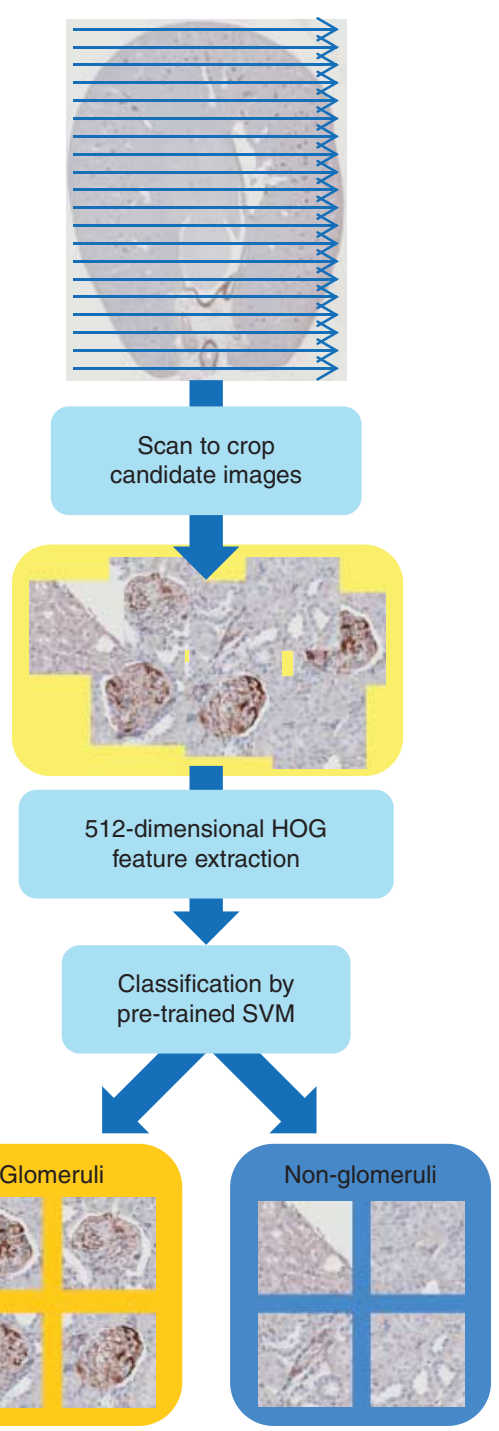

B

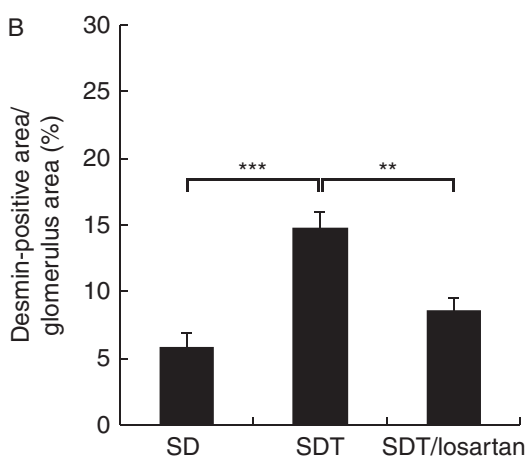

http://joe.endocrinology-journals.org DOI: 10.1530/JOE-14-0164 used as an input vector for a pretrained binary classifier, a linear SVM (Schölkopf \& Smola 2002, Shawe-Taylor \& Cristianini 2004). As a result, the method automatically detected glomeruli in a whole-kidney section and subsequently quantified the glomerular-immunopositive area in the sub-images using standard image processing.

Automated, quantitative image analysis showed a significant increase in glomerular desmin-immunopositive area in SDT rats compared with SD rats (Fig. 3B). Treatment with losartan significantly decreased glomerular desmin-immunopositive area in SDT rats. This result indicates that glomerular desmin immunoreactivity could be a more sensitive marker for drug efficacy than urinary albumin in studies of rat diabetic nephropathy.

\section{Heterogeneity of glomerular desmin immunoreactivity in SDT rat kidneys}

It has been reported that podocyte injury, evaluated by desmin immunoreactivity, occurred in juxtamedullary glomeruli before superficial glomeruli in type 2 diabetic OLETF rats (Sofue et al. 2012). The authors also showed that treatment with olmesartan, another ARB, prevented the increase in glomerular desmin-immunopositive area in both superficial and juxtamedullary glomeruli. In this study, we studied desmin-positive areas in superficial and juxtamedullary glomeruli in diabetic SDT rats using the new computational method. The intrarenal distribution of glomerular injury was established by labeling glomeruli as members of one of two classes defined by the value of desmin-immunopositive area $(<20 \%$ and $\geq 20 \%$ of glomerular area) on microscopic images (Fig. 4). Similar to OLETF rats, SDT rat kidneys showed a larger desminimmunopositive area in juxtamedullary glomeruli than in superficial ones (Fig. 5). Treatment with losartan prevented the increase in glomerular desmin-immunopositive area in both superficial and juxtamedullary glomeruli.

\section{Figure 3}

Automated, quantitative image analysis of glomerular desmin-immunopositive area in SD and SDT rat kidneys. (A) Workflow of the developed, automatic computational image analysis method. A whole-kidney section image was scanned with a detection window, and candidate sub-images were cropped. Using the extracted 512-dimensional HOG feature vector, the pretrained linear SVM algorithm classified the candidate cropped sub-images containing glomeruli and those without glomerulus. As a result, the algorithm automatically detected glomeruli in a whole kidney section and quantified the glomerular desmin-immunopositive area thereafter. (B) Glomerular desmin-immunopositive area of SD and SDT (control and losartan-treated) rats at 24 weeks of age is shown. Statistically significant differences are indicated by $* * P<0.01$ and $* * * P<0.001$.

Published by Bioscientifica Ltd. 

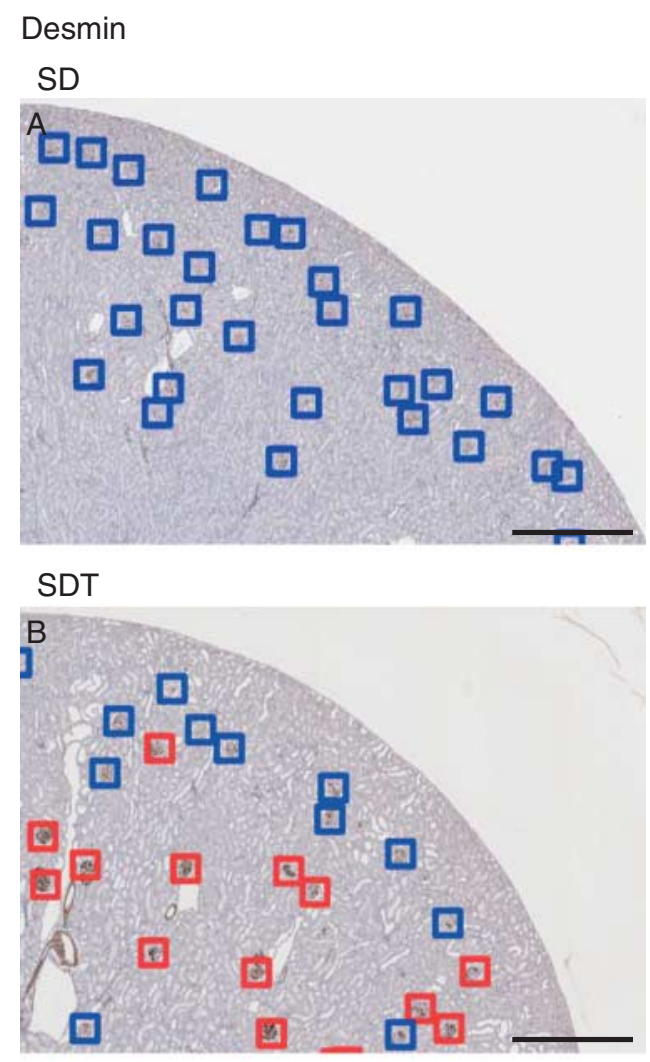

SDT/losartan

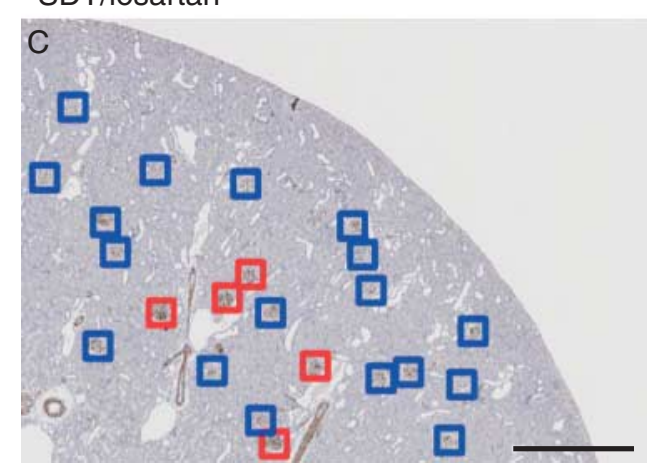

Figure 4

Distribution of glomerular injuries denoted by desmin immunostaining in SD (A), SDT (B), and losartan-treated SDT rats (C) at 24 weeks of age. The computational method automatically recognized and assigned glomeruli to one of two classes according to the desmin-immunopositive area: $<20 \%$ (blue) and $\geq 20 \%$ (red) of glomerular area. Scale bars: $1 \mathrm{~mm}$.

\section{Heterogeneity of glomerular desmin immunoreactivity in PAN-induced nephropathy}

To investigate whether the heterogeneity of glomerular injury within a kidney was limited to diabetic nephropathy, we also quantitatively studied glomerular desmin immunoreactivity of rats with PAN-induced nephropathy.
The PAN model is well established and characterized by proteinuria and selective podocyte injury (Zou et al. 2006, Herrmann et al. 2012). As illustrated in Fig. 6, we labeled glomeruli on microscopic images as belonging to one of two classes by virtue of their desmin-immunopositive area: $<40 \%$ and $\geq 40 \%$. PAN rat glomeruli displayed a strong increase in desmin-positive area (Figs 6 and 7). On day 4 after PAN injection, glomeruli located in the juxtamedullary region displayed almost maximal desmin immunostaining, while those located at the superficial cortex showed a lesser increase in desmin immunostaining. At this stage of damage, the results were consistent with those for diabetic SDT rats. On day 7 after PAN injection, both superficial and juxtamedullary glomeruli displayed maximal desmin immunoreactivity. Thus, the podocyte injury marker desmin was increased in juxtamedullary glomeruli before superficial ones in PANinduced toxic nephropathy as well as in diabetic nephropathy.
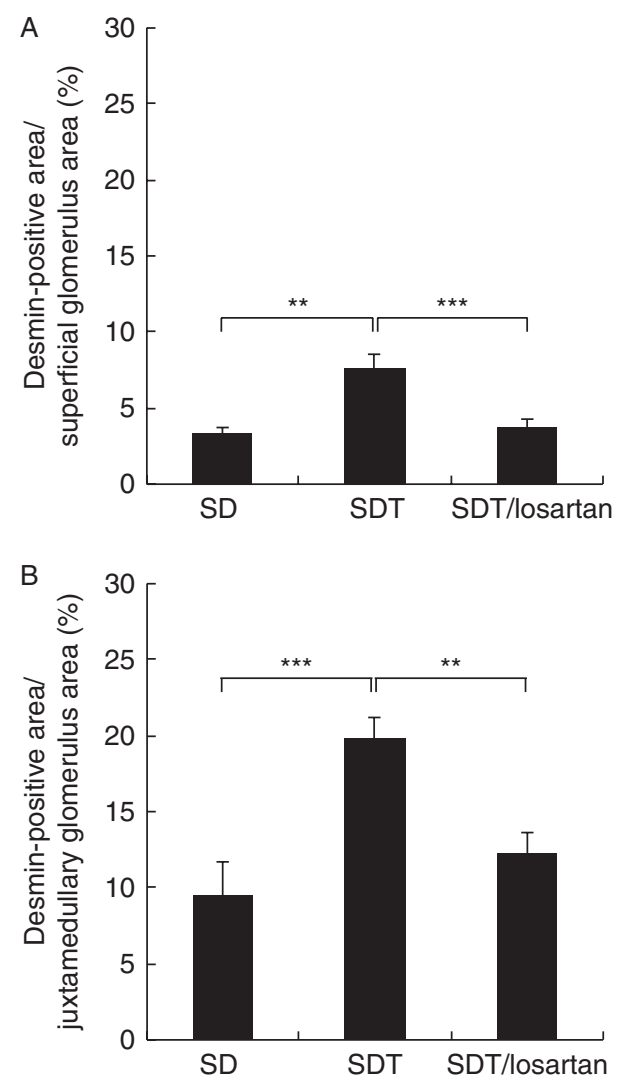

Figure 5

Automated, quantitative image analysis of superficial (A) and juxtamedullary (B) glomeruli in desmin-immunostained kidneys from SD and SDT (control and losartan-treated) rats at 24 weeks of age. Statistically significant differences are indicated by $* * P<0.01$ and $* * * P<0.001$. 


\section{Desmin}

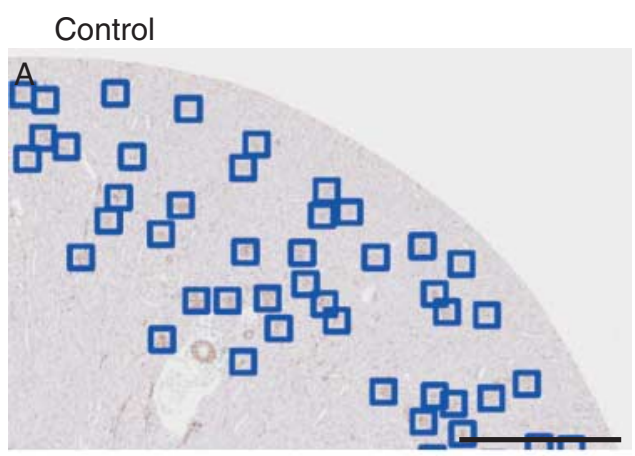

PAN day 4

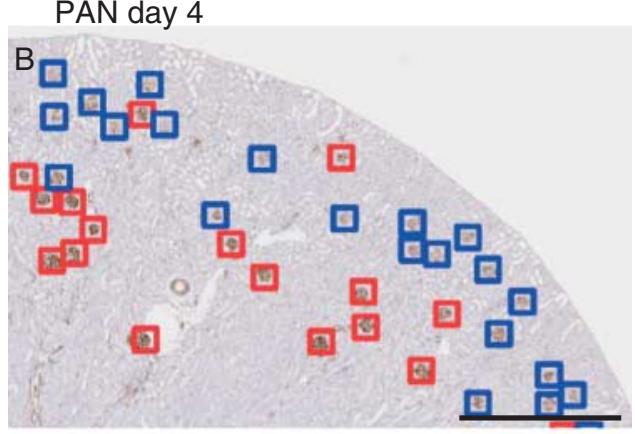

PAN day 7

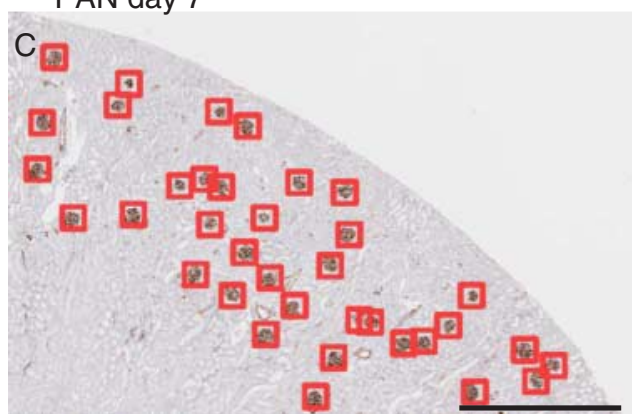

Figure 6

Distribution of glomerular injury denoted by desmin immunostaining in control (A) and PAN model rats on day 4 (B) and day 7 (C) after PAN injection. The computational method automatically recognized and labeled glomeruli as members of one of two classes according to desminimmunopositive area: $<40 \%$ (blue) and $\geq 40 \%$ (red). Scale bars: $1 \mathrm{~mm}$.

\section{Discussion}

Image analysis of glomeruli in kidney specimens is important for quantitative elucidation of the pathophysiology of diabetic nephropathy, both in the field of preclinical drug efficacy studies as well as in clinical pathology (Herrmann et al. 2012, Sofue et al. 2012). For the quantification of the glomerular desmin-positive area, distinguishing glomeruli from other kidney structures is still challenging. A manually assisted approach, where each glomerulus border is traced by hand, will provide accurate results, but is very time-consuming. Given that glomerular injury could be heterogeneous (Sofue et al. 2012; Figs 4, 5, 6 and 7), it is mandatory that as many glomeruli as possible should be quantified, far beyond the capacity of manual tracing. Automated recognition of
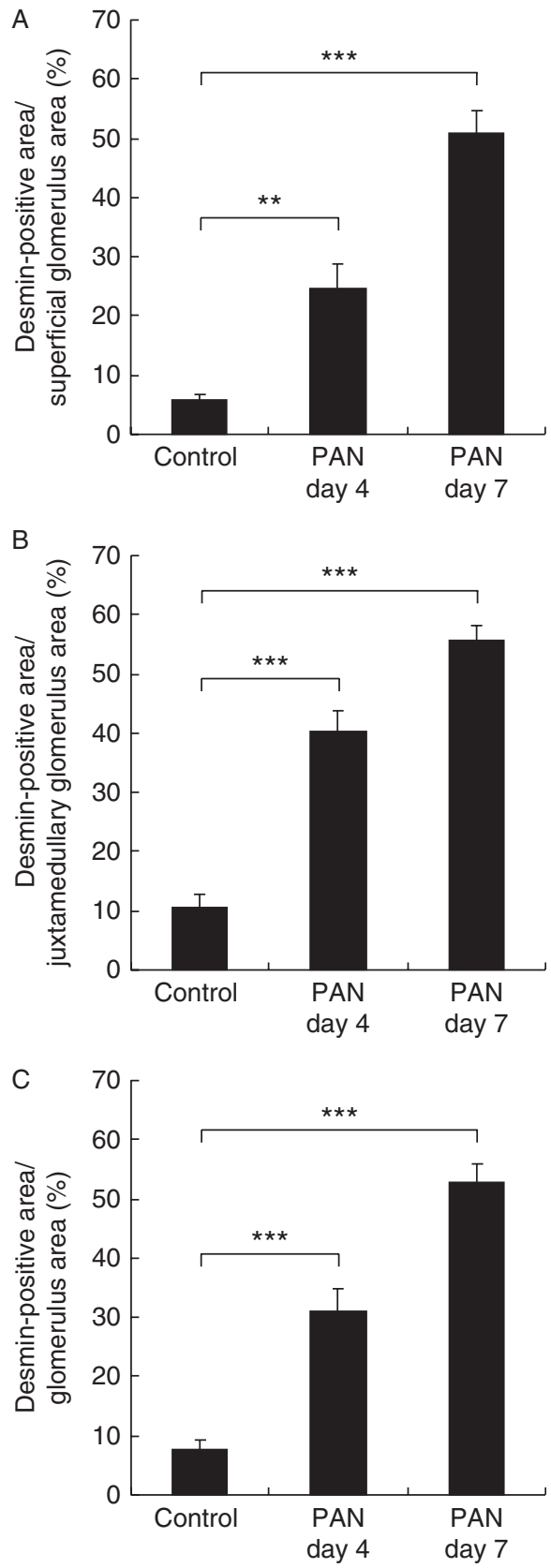

Figure 7

Automated, quantitative image analysis of superficial (A), juxtamedullary (B), and whole (C) glomeruli in desmin-immunostained kidneys from control and PAN model rats. Glomerular desmin-positive area from control and PAN nephropathy kidneys on days 4 and 7 is shown. Statistically significant differences are indicated by $* * P<0.01$ and $* * * P<0.001$. 
glomeruli is challenging for most commercial image analysis software, including tissue pattern recognition software (Kakimoto et al. 2013), because they are based on color feature information of tissue structures for recognition. In this study, we have developed a novel method, applying the state-of-the-art computational method used in face- and car-recognition systems, to detect glomeruli. The method enabled automatic recognition and quantification of the glomerular desmin-immunopositive area, eliminating the need for laborious manual quantification.

To establish whether the method may facilitate studies of diabetic nephropathy, we have demonstrated a specific application to examine glomerular injury and drug efficacy in diabetic SDT rats. The SDT rat strain is a new model for non-obese type 2 diabetes with impaired insulin secretion and subsequent diabetic complications (Shinohara et al. 2000, Ohta et al. 2007, Sasase et al. 2013). As SDT rats develop diabetic complications that reflect human type 2 diabetes, they are increasingly regarded as a suitable model for preclinical drug efficacy studies of diabetic complications, including diabetic nephropathy. However, early glomerular podocyte injury has not been sufficiently studied in SDT rats. In this study, we have demonstrated that desmin could serve as a sensitive and valuable marker for early glomerular damage in SDT rats. Indeed, losartan treatment significantly lowered glomerular desmin immunoreactivity, while urinary albumin was not significantly different.

Involvement of the renin-angiotensin system in the pathogenesis of diabetic nephropathy and the therapeutic effect of agents blocking the pathway have been extensively studied (Nishiyama et al. 2008, Sofue et al. 2012). ARBs reportedly had beneficial effects on diabetic nephropathy in type 2 diabetes patients (Brenner et al. 2001). However, the effect of early ARB treatment on SDT rat diabetic nephropathy was not known. In this study, we have shown that early treatment with an ARB, losartan, prevented the onset of glomerular injury in SDT rats. Losartan treatment significantly lowered glomerular desmin immunoreactivity, indicating that ARB treatment from the early stages could be an effective strategy for preventing the development of diabetic nephropathy later in life. Although losartan did not affect blood glucose level in SDT rats, losartan treatment significantly decreased glomerular damage. The direct ameliorative effect of losartan was possibly due to the inhibition of the local renin-angiotensin system in SDT kidneys (Nishiyama et al. 2008).

It has been reported that podocyte injury occurred in juxtamedullary glomeruli before superficial glomeruli in type 2 diabetic OLETF rats (Sofue et al. 2012). Hypertensive glomerular damage also reportedly occurred first and more severely in the juxtamedullary glomeruli compared with superficial ones in spontaneously hypertensive rats (Iversen et al. 1998). In this study, we showed that diabetic SDT rat glomerular injury was more predominant in the juxtamedullary region than in the superficial cortex. The novel computational method was quite helpful to clearly reveal the distribution of glomerular injuries within a kidney. Interestingly, we also found that PAN toxicity-induced podocyte injury also occurred at juxtamedullary glomeruli before superficial ones.

Juxtamedullary glomeruli are located deep in the renal cortex and their afferent arterioles arise from either the initial segments of interlobular arteries or directly from arcuate arteries, while the afferent arterioles of superficial glomeruli branch off from distal segments of interlobular arteries. The short distances of juxtamedullary nephrons from large arcuate arteries impose substantially higher feeding pressure load on these nephrons (Ito et al. 2009). This, in turn, would supposedly make them more vulnerable to various stresses including diabetes, hypertension, and even toxicity compared with superficial nephrons. Some differences in the mechanism of autoregulation in superficial and juxtamedullary nephrons, such as actions angiotensin II, might contribute to the heterogeneity of glomerular injuries as well.

In conclusion, we demonstrated early glomerular injury of SDT rats by utilizing desmin as a sensitive podocyte injury marker. The state-of-the-art automated image analysis method enabled quantitative and objective analysis of the ameliorative effect of early losartan treatment on glomerular injury. We also found that glomerular injury was more severe in the juxtamedullary region than in the superficial cortex, not only in diabetic SDT rat nephropathy, but also in PAN toxicity-induced nephropathy. Thus, application of the automated image analysis method to glomerular injury is feasible and may facilitate further elucidation of the pathogenesis of diabetic nephropathy and discovery of new drugs.

Supplementary data

This is linked to the online version of the paper at http://dx.doi.org/10.1530/ JOE-14-0164.

Declaration of interest

T K, K O, M K, TI, K F, M N, A F, and H U work for Mitsubishi Tanabe Pharma Corporation.

Published by Bioscientifica Ltd. 


\section{Funding}

This research did not receive any specific grant from any funding agency in the public, commercial or not-for-profit sector.

\section{Acknowledgements}

The authors thank Ms Yasuko Ogawa for her excellent technical assistance. They also thank Prof. Dr Bernhard F Becker (Ludwig-Maximilians-University, Munich) for restyling our English.

\section{References}

Brenner BM, Cooper ME, de Zeeuw D, Keane WF, Mitch WE, Parving HH, Remuzzi G, Snapinn SM, Zhang Z, Shahinfar S et al. 2001 Effects of losartan on renal and cardiovascular outcomes in patients with type 2 diabetes and nephropathy. New England Journal of Medicine $\mathbf{3 4 5}$ 861-869. (doi:10.1056/NEJMoa011161)

Dalal N \& Triggs B 2005 Histograms of oriented gradients for human detection. Proceedings of the 2005 IEEE Computer Society Conference on Computer Vision and Pattern Recognition, Volume 1 of CVPR 2005 IEEE Computer Society, San Diego, CA, USA. pp 886-893. (doi:10.1109/ CVPR.2005.177)

Floege J, Alpers CE, Sage EH, Pritzl P, Gordon K, Johnson RJ \& Couser WG 1992 Markers of complement-dependent and complement-independent glomerular visceral epithelial cell injury in vivo. Expression of antiadhesive proteins and cytoskeletal changes. Laboratory Investigation 67 486-497.

Fujii H, Kono K, Nakai K, Goto S, Komaba H, Hamada Y, Shinohara M, Kitazawa R, Kitazawa S \& Fukagawa M 2010 Oxidative and nitrosative stress and progression of diabetic nephropathy in type 2 diabetes. American Journal of Nephrology 31 342-352. (doi:10.1159/000297290)

Herrmann A, Tozzo E \& Funk J 2012 Semi-automated quantitative image analysis of podocyte desmin immunoreactivity as a sensitive marker for acute glomerular damage in the rat puromycin aminonucleoside nephrosis (PAN) model. Experimental and Toxicologic Pathology 64 45-49. (doi:10.1016/j.etp.2010.06.004)

Hoshi S, Shu Y, Yoshida F, Inagaki T, Sonoda J, Watanabe T, Nomoto K \& Nagata M 2002 Podocyte injury promotes progressive nephropathy in Zucker diabetic fatty rats. Laboratory Investigation 82 25-35. (doi:10.1038/labinvest.3780392)

Ito S, Nagasawa T, Abe M \& Mori T 2009 Strain vessel hypothesis: a viewpoint for linkage of albuminuria and cerebro-cardiovascular risk. Hypertension Research 32 115-121. (doi:10.1038/hr.2008.27)

Iversen BM, Amann K, Kvam FI, Wang X \& Ofstad J 1998 Increased glomerular capillary pressure and size mediate glomerulosclerosis in
SHR juxtamedullary cortex. American Journal of Physiology 274 F365-F373.

Kakimoto T, Kimata H, Iwasaki S, Fukunari A \& Utsumi H 2013 Automated recognition and quantification of pancreatic islets in Zucker diabetic fatty rats treated with exendin-4. Journal of Endocrinology 216 13-20. (doi:10.1530/JOE-12-0456)

Kim J, Shon E, Kim CS \& Kim JS 2012 Renal podocyte injury in a rat model of type 2 diabetes is prevented by metformin. Experimental Diabetes Research 2012 210821. (doi:10.1155/2012/210821)

Nishiyama A, Nakagawa T, Kobori H, Nagai Y, Okada N, Konishi Y, Morikawa T, Okumura M, Meda I, Kiyomoto H et al. 2008 Strict angiotensin blockade prevents the augmentation of intrarenal angiotensin II and podocyte abnormalities in type 2 diabetic rats with microalbuminuria. Journal of Hypertension 26 1849-1859. (doi:10.1097/HJH.0b013e3283060efa)

Ohta T, Matsui K, Miyajima K, Sasase T, Masuyama T, Shoda T, Koizumi H, Shinohara M \& Matsushita M 2007 Effect of insulin therapy on renal changes in spontaneously diabetic Torii rats. Experimental Animals 56 355-362. (doi:10.1538/expanim.56.355)

Sasase T, Ohta T, Masuyama T, Yokoi N, Kakehashi A \& Shinohara M 2013 The spontaneously diabetic Torii rat: an animal model of nonobese type 2 diabetes with severe diabetic complications. Journal of Diabetes Research 2013 976209. (doi:10.1155/2013/976209)

Schölkopf B \& Smola AJ 2002 Learning with Kernels. Cambridge, MA: MIT Press.

Shawe-Taylor J \& Cristianini N 2004 Kernel Methods for Pattern Analysis. Cambridge: Cambridge University Press.

Shinohara M, Masuyama T, Shoda T, Takahashi T, Katsuda Y, Komeda K, Kuroki M, Kakehashi A \& Kanazawa Y 2000 A new spontaneously diabetic non-obese Torii rat strain with severe ocular complications. International Journal of Experimental Diabetes Research 1 89-100. (doi:10.1155/EDR.2000.89)

Sofue T, Kiyomoto H, Kobori H, Urushihara M, Nishijima Y, Kaifu K, Hara T, Matsumoto S, Ichimura A, Ohsaki H et al. 2012 Early treatment with olmesartan prevents juxtamedullary glomerular podocyte injury and the onset of microalbuminuria in type 2 diabetic rats. American Journal of Hypertension 25 604-611. (doi:10.1038/ajh. 2012.1)

Wiggins RC 2007 The spectrum of podocytopathies: a unifying view of glomerular diseases. Kidney International 71 1205-1214. (doi:10.1038/ sj.ki.5002222)

Williams ME 2010 Diabetic CKD/ESRD 2010: a progress report? Seminars in Dialysis 23 129-133. (doi:10.1111/j.1525-139X.2009.00698.x)

Zou J, Yaoita E, Watanabe Y, Yoshida Y, Nameta M, Li H, Qu Z \& Yamamoto T 2006 Upregulation of nestin, vimentin, and desmin in rat podocytes in response to injury. Virchows Archiv: an International Journal of Pathology 448 485-492. (doi:10.1007/s00428-005-0134-9)

Received in final form 24 April 2014

Accepted 28 April 2014

Accepted Preprint published online 29 April 2014 http://joe.endocrinology-journals.org DOI: 10.1530/JOE-14-0164
C 2014 Society for Endocrinology Printed in Great Britain
Published by Bioscientifica Ltd. 